\title{
The effect of exercise therapy, dry needling, and nonfunctional electrical stimulation on radicular pain: a case report
}

\author{
Lida Hosseini', Ardalan Shariat ${ }^{1, *}$, Maryam Selk Ghaffari', Roshanak Honarpishe ${ }^{2}$, Joshua A. Cleland ${ }^{3}$ \\ ${ }^{1}$ Sports Medicine Research Center, Neuroscience Institute, Tehran University of Medical Sciences, Tehran, Iran \\ ${ }^{2}$ Department of Physiotherapy, School of Rehabilitation, Tehran University of Medical Sciences, Tehran, Iran \\ ${ }^{3}$ Franklin Pierce University, Manchester, NH, USA
}

A 43-year-old male, office worker with history of chronic radicular low back pain radiating into the left leg was admitted to a sports medicine research center, neuroscience institute. During the past year, he visited a physiotherapist and orthopedic experts. Magnetic resonance imaging revealed a protruded disc at L4-5 level. Additionally, electromyography indicated that there was bilateral moderate irritation at the L5-S1 root. We designed a management package including exercise therapy, dry needling, and nonfunctional electrical stimulation for four sessions. Outcomes included pain intensity, pain with lumbar flexion, with the nu- merical rating scale (NRS), visual analogue scale (VAS), and function measured with the Oswestry Disability Index before and after the intervention. After 4 treatment sessions, the patient reported a reduction in pain intensity from a 9 to 2 on the NRS and from 90 to 30 on the VAS. In addition, the patient was able to perform lumbar flexion fully without pain.

Keywords: Therapy, Exercise, Low back pain, Neurorehabilitation

\section{INTRODUCTION}

Low back pain (LBP) is a major health burden which affects the majority of individuals in the world (Tüzün et al., 2017). It has been estimated that about $60 \%-80 \%$ of people experience LBP in their lifetime (Liu et al., 2018; Tüzün et al., 2017). This results in considerable implications on disability, quality of life, work status, and results in substantial economic burden for society (Liu et al., 2018).

Numerous management strategies exist for the treatment of chronic LBP. The initial treatment options are typically noninvasive and include; pharmacological medications, manual therapy, and exercise therapy (Jorgensen et al., 2018). Exercise therapy is one of the most common treatment approaches for the management of LBP (Aboagye et al., 2015). Studies have shown that exercise therapy can improve function and activities of daily living by improving flexibility, strength and endurance (Nguyen et al., 2017; Shariat et al., 2017; Takahashi et al., 2017).

Dry needling is another technique used to manage trigger points (TPs) in muscles such as the gluteal muscle which have been shown to be prevalent in individuals with lumbosacral radiculopathy (Adelmanesh et al., 2015). Dry needling has been shown to increase pressure pain threshold and range of motion (ROM), decrease muscle tone, and decrease pain as measured as a reduction in sensitivity of TPs (Shariat et al., 2018). In the needling technique, a fine needle is used to penetrate the skin, subcutaneous tissues, and muscle without using anesthetics (Gattie et al., 2017). However there exists controversy as to the clinical effectiveness of dry needling compared to other management approaches (Tough et al., 2009). Despite this it is still recommended for use in the management of chronic LBP (Furlan et al., 2005).

Nonfunctional electrical stimulation (ES) is a therapeutic mo-
${ }^{\star}$ Corresponding author: Ardalan Shariat (iD https://orcid.org/0000-0002-1877-4747 Sports Medicine Research Center, Neuroscience Institute, Tehran University of Medical Sciences, Tehran, Iran

Tel: +98-9398614772, Fax: +98-2188630227, E-mail: ardalansh2002@gmail.com Received: July 28, 2018 / Accepted: August 27, 2018
This is an Open Access article distributed under the terms of the Creative Commons Attribution Non-Commercial License (http://creativecommons.org/licenses/by-nc/4.0/) which permits unrestricted non-commercial use, distribution, and reproduction in any medium, provided the original work is properly cited. 
dality where nerves are stimulated with electrical current via surface electrodes to stimulate muscular contraction to improve strength and physical performance (Bauer et al., 2015). This method has been widely used for the management of nonacute LBP (Glaser et al., 2001). It is plausible this method has potential to reduce pain in patients with LBP by strengthening trunk muscles, similar to exercise.

This case report describes the outcomes of a patient with low back and radicular pain receiving a treatment package including exercise therapy, dry needling and nonfunctional ES. To the best of our knowledge no reports in the literature could be identified using this multimodal treatment approach.

\section{MATERIALS AND METHODS}

\section{Case history}

A 43-year-old male, office worker was admitted to a sports medicine research center, neuroscience institute, with severe LBP with radiculopathy into the left lower extremity. The patient reported numbness and pain in his left leg of approximately a 1-year duration but reported the pain had worsened over the past 2 months. His medical history was unremarkable. The patient spent up to $9 \mathrm{hr}$ per day sitting which increased the level of the lumbar pain which subsequently spread to the left hip, buttock and lateral side of the left thigh, with numbness and paresthesia into the lateral side of the left leg. The patient noted the pain increased by sitting and improved by standing.

During the past year, he had visited numerous healthcare providers including an orthopedic surgeon and a physiotherapist. The physician performed magnetic resonance imaging (MRI) which revealed a protruded disc at the L4-5 level (Fig. 1). Additionally, electromyography results indicated bilateral moderate irritation at the L5-S1 root.

Following the imaging, he received three days of complete bed rest, eight sessions of electro-acupuncture (three sessions per week), and was instructed to use ice 4 times per day. He reported this management approach resulted in a worsening of his symptom. At that time, he was ordered to remain on bed rest for 21 days, which also included ten physiotherapy sessions (four sessions in first week, three sessions in second week, two sessions in third week and one session in last week) and six hydrotherapy sessions. A follow-up MRI revealed there was no change in the disc protrusion (Fig. 2).

\section{Evaluation}

When he visited our clinic, the physical examination indicated painful but complete active lumbar flexion. Also, the straight leg rise test was positive $\left(35^{\circ}-70^{\circ}\right)$ only on the left side and TPs were detected in the gluteus medius muscle on the painful side. In addition, the patient had difficulty walking on his heel. Deep ten-

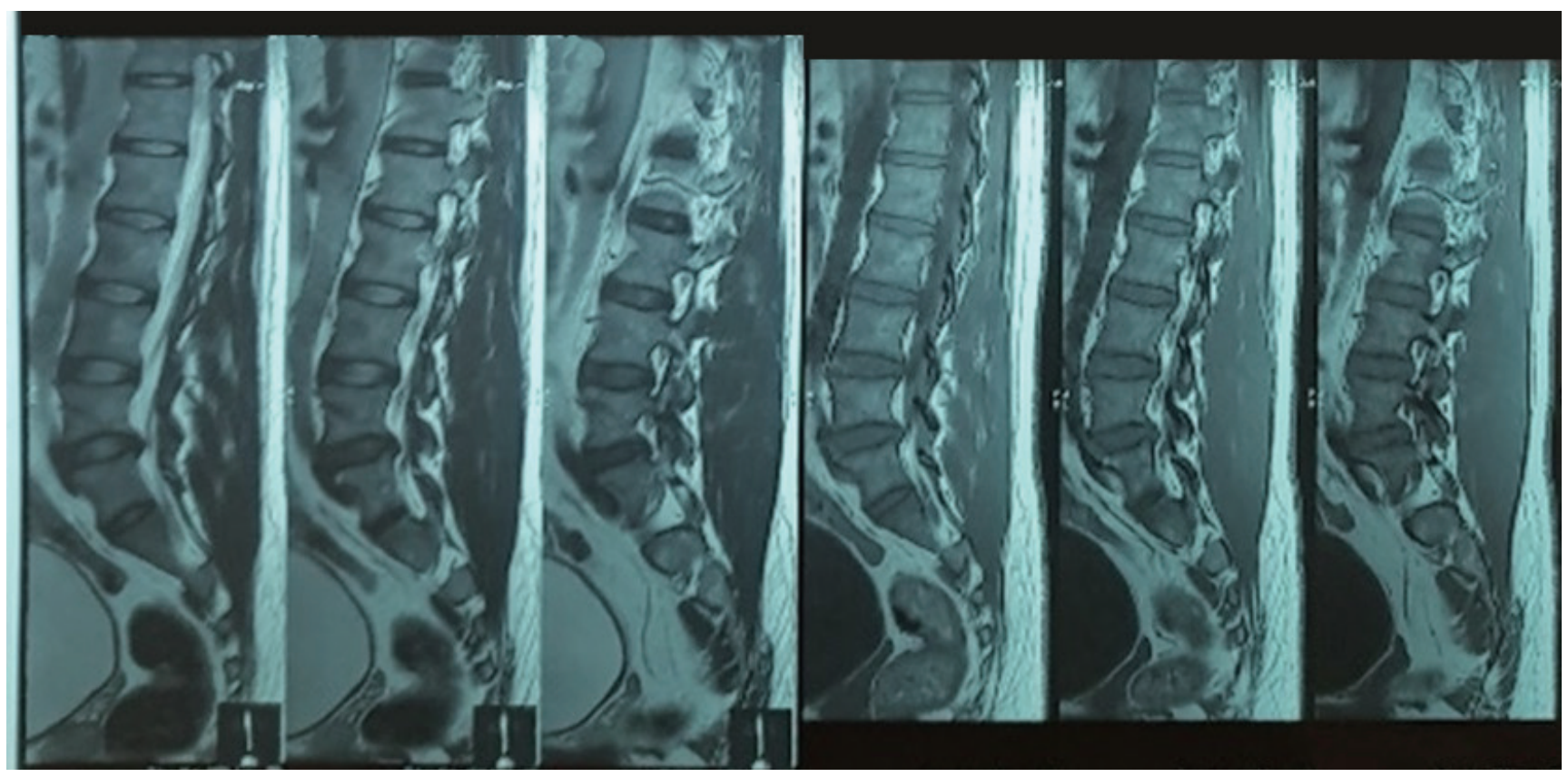

Fig. 1. T2-weighted magnetic resonance image (MRI). Sagittal view. First MRI revealed a broad base protruded disc at L4-5 disc level. 

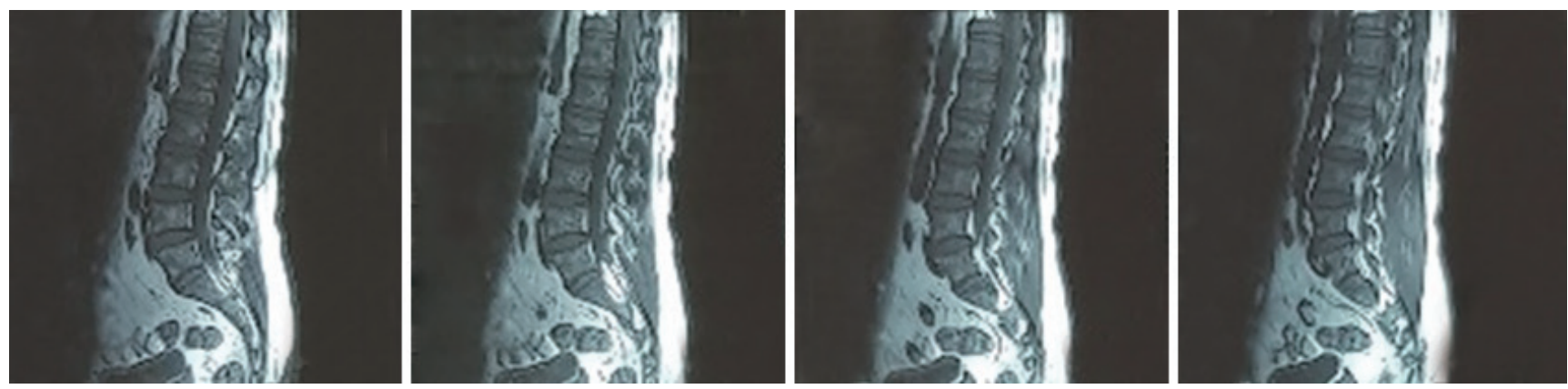

Fig. 2. Second MRI. T2-weighted magnetic resonance image (MRI). Sagittal view. MRI revealed a broad base protruded disc at L4-5 disc level and no significant changes were detected according to previous MRI.

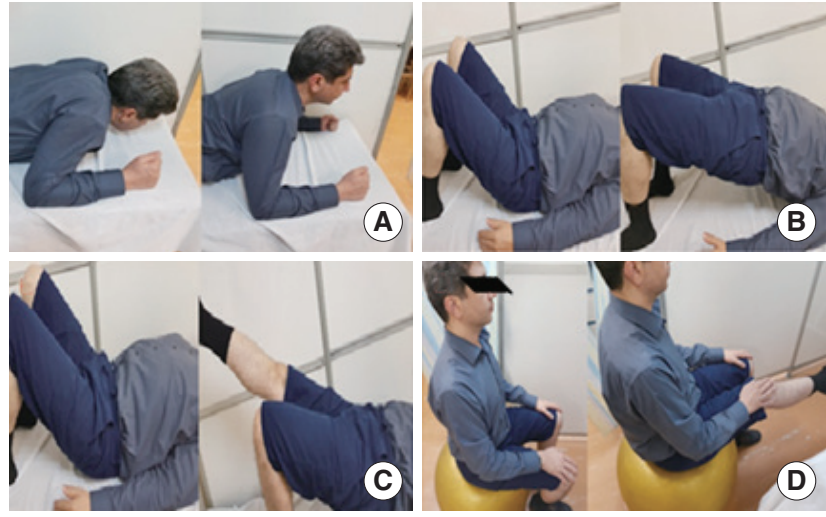

Fig. 3. Package of exercise therapy.

don reflexes were $2+$ and no sensory defects were identified. Furthermore, pain intensity was assessed by using the numeric rating scale (NRS) and visual analogue scale (VAS) scale. The NRS has been shown to have acceptable levels of reliability and validity (Bijur et al., 2003; Welsh, 2016). We asked the patients to rate his pain on a 0 to 10 scale. The patient reported he was currently at a "9."

The VAS is in a $10-\mathrm{cm}$ horizontal line on which the patent marks their level of pain. Previous studies have shown the VAS to possess acceptable reliability and validity (Phan et al., 2012; Welsh, 2016). The patient scored the VAS at a $90 \mathrm{~mm}$. Functional disability was evaluated by using the Oswestry disability score, which was a $60 \%$ before the intervention. Reliability and content validity of Oswestry questionnaire are reported by Fisher and Johnston (1997).

\section{Intervention}

We designed a specific package including exercise therapy (Fig. 3), dry needling (Fig. 4), and nonfunctional ES (Fig. 5) over four sessions (Table 1). Dry needling was directed at the gluteus medi-

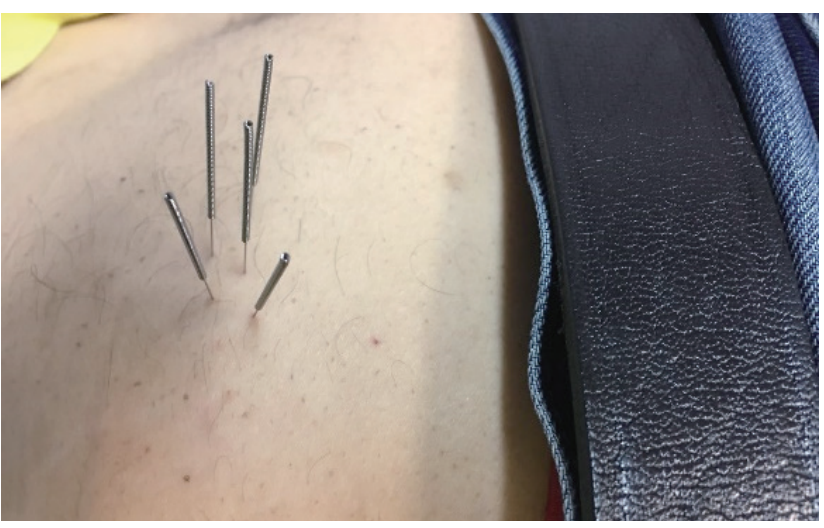

Fig. 4. Location of dry needling.

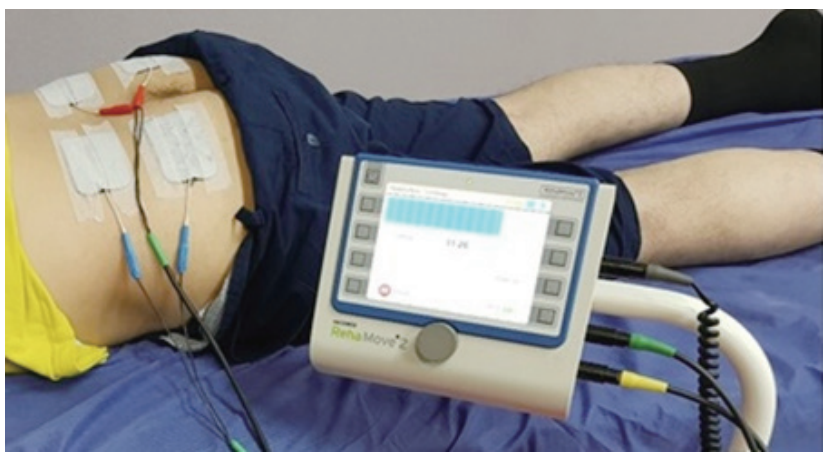

Fig. 5. Nonfunctional electrical stimulation.

us muscle to release TPs (Fig. 4).

After the final session, we again measured the outcomes of pain intensity with NRS, VAS, and flexion ROM (as it was full in the beginning, but painful). At the final session, the pain had centralized to the low back and the no longer reported experiencing pain or numbness in the left lower extremity. At this time, the measurements of pain were 2 points for the NRS and $30 \mathrm{~mm}$ on the VAS. Additionally, the patient was able to perform full lumbar flexion 
Table 1. Package of treatment

\begin{tabular}{|c|c|c|}
\hline Sessions & Protocols & Characterize and duration \\
\hline \multirow[t]{3}{*}{ Sessions 1-4 } & $\begin{array}{l}\text { (1) Exercise therapy* } \\
\text { (Fig. 3) }\end{array}$ & $\begin{array}{l}\text { A. Extension type+activation transverse abdominis exercise } \\
\text { B. Bridging exercise } \\
\text { C. Bridging with single leg exercise } \\
\text { D. Core stability exercises }\end{array}$ \\
\hline & (2) Dry needling (Fig. 4) & $\begin{array}{l}\text { Five stainless steel dry needles (size, } 60 \times 25 \text { mm; SMC, Seoul, Korea) were inserted into the left in the gluteus medius muscle and } \\
\text { were moved in and out for } 30 \mathrm{~min} \text {. }\end{array}$ \\
\hline & $\begin{array}{l}\text { (3) Nonfunctional electric } \\
\text { stimulation (Fig. 5) }\end{array}$ & $\begin{array}{l}\text { Four electric stimulation pads were placed around the treatment area (lumbar erector spine bilateral and gluteal muscles bilateral). } \\
\text { The frequency of simulation was } 50 \mathrm{~Hz} \text {, pulse duration was } 450 \mu \mathrm{sec} \text {, amplitude was } 42 \mathrm{~mA} \text { and interpulse interval was } 0.6 \text { sec. } \\
\text { the total time of stimulation was } 15 \mathrm{~min} \text {. Motor responses were achieved as contraction produced under the electrodes. }\end{array}$ \\
\hline
\end{tabular}

*The first session of exercises was performed in the clinic and we asked the patient to perform the second set of exercises at home during the afternoon.

Table 2. Pain intensity and range of motion before and after the intervention

\begin{tabular}{lcc}
\hline Variable & Preintervention & Postintervention \\
\hline Numeric rating scale & $9 / 10$ & $2 / 10$ \\
Visual analogue scale & $90 / 100$ & $30 / 100$ \\
$\begin{array}{c}\text { Active range of motion } \\
\text { (lumbar flexion) }\end{array}$ & $\begin{array}{c}\text { Full range of motion and } \\
\text { painful }\end{array}$ & $\begin{array}{c}\text { Full range of motion and } \\
\text { pain-free }\end{array}$ \\
\hline
\end{tabular}

pain free. Furthermore, straight leg raise on the left was negative and there were no TPs detected in the piriformis muscle. Oswestry disability scores was $20 \%$. See details of results in Table 2.

The approval of the Research Ethics Committee of the Sports Medicine Research Center, Iran, was obtained on their monthly meeting on 15 October 2017 (approval number: IR.TUMS.REC. 2871).

\section{RESULTS AND DISCUSSION}

LBP is one of the main reasons individuals miss days from work and results in substantial disability for office workers (Shariat et al., 2016). There are several management strategies for individuals with LBP that range from noninvasive to invasive procedures (Coulombe et al., 2017). There exist many different approaches to exercise therapy and a variety of approaches have been shown to reduce pain and improve ROM in individuals with LBP. For example, 12 weeks of core stabilization exercise and general exercise are effective interventions for young male patients with lumbar disc herniation (Ye et al., 2015). Shariat et al. (2017) demonstrated the long term effect of exercise were more effective than ergonomic modification on neck, shoulder, and LBP among office workers. Although studies have shown that exercise therapy results in the reduction of LBP no general consensus exists regarding the most efficacious approach. (Jorgensen et al., 2018; Nguyen et al., 2017). However, it has been shown that core stability exercises may result in better coping strategy for dealing with the pain. In addition, $17 \%$ increases in plasma serotonin levels have been reported immediately after $30 \mathrm{~min}$ of stabilization exercise, which may be related to the positive effects of the exercise approach used with the current patient after only four sessions (Ganiyu and Gujba, 2015).

Dry needling is another technique that has been used for reducing LBP by deactivating myofascial trigger points. It has been demonstrated to be effective in conjunction with other therapies for chronic LBP (Nguyen et al., 2017). Dry needling can mechanically disrupt sensory or motor components of nerve endings that contribute to abnormal elements of muscle contractions. The temporary damage to the muscle fibers created by the needle may cause the release of local intracellular potassium which prevents depolarization of nerve fibers. In addition, other plausible theories exist for potential mechanisms of action including the release of local endorphins and the gate control theory of pain (Tüzün et al., 2017). Nevertheless, a systematic review and meta-analysis performed by on the effects of dry needling on neck pain concluded that there is a low-quality to moderate quality evidence for the effectiveness of this method on neck pain (Gattie et al., 2017). Furthermore, a systematic review by Liu et al. (2018) found needling reduced the intensity of pain in individuals with LBP, however the impact on function reamins unclear (Liu et al., 2018).

Several studies have assessed the effects of neuromuscular ES with the addition of exercise on musculoskeletal spine pain. Hicks et al. (2016) found improvements in physical function in elderly individuals with chronic LBP. In addition, it has been showed ES stimulates hip and trunk muscles and decrease pain, but the duration of these findings was short-term only (Triolo et al., 2009; Yang et al., 2009).

Different combinations of conservative management strategies are used for reducing pain and improving function in individuals with chronic disc herniation but it is difficult to ascertain which approach is more effective. 
We report the outcomes of a patient treated with a short-term multimodal approach including exercise therapy, dry needling, and nonfunctional ES to reduce pain in and increase ROM in a patient with LBP. During the course of four treatment sessions this individual experienced clinically meaningful improvements in pain and ROM. However, we cannot infer a cause and effect relationship from a case study. In our view, a combination of these three treatments may have been the key to reducing the radicular pain and we suggest multimodal approaches including exercise therapy, dry needling and nonfunctional ES instead be combined for the management of this condition. Future randomized clinical trials should be performed to determine if the treatment approach used in the current case study is superior to other approaches.

\section{CONFLICT OF INTEREST}

No potential conflict of interest relevant to this article was reported.

\section{REFERENCES}

Aboagye E, Karlsson ML, Hagberg J, Jensen I. Cost-effectiveness of early interventions for non-specific low back pain: a randomized controlled study investigating medical yoga, exercise therapy and self-care advice. J Rehabil Med 2015;47:167-173.

Adelmanesh F, Jalali A, Jazayeri Shooshtari SM, Raissi GR, Ketabchi SM, Shir $Y$. Is there an association between lumbosacral radiculopathy and painful gluteal trigger points?: a cross-sectional study. Am J Phys Med Rehabil 2015;94:784-791.

Bauer P, Krewer C, Golaszewski S, Koenig E, Müller F. Functional electrical stimulation-assisted active cycling-therapeutic effects in patients with hemiparesis from 7 days to 6 months after stroke: a randomized controlled pilot study. Arch Phys Med Rehabil 2015;96:188-196.

Bijur PE, Latimer CT, Gallagher EJ. Validation of a verbally administered numerical rating scale of acute pain for use in the emergency department. Acad Emerg Med 2003;10:390-392.

Coulombe BJ, Games KE, Neil ER, Eberman LE. Core stability exercise versus general exercise for chronic low back pain. J Athl Train 2017;52: 71-72.

Fisher K, Johnston M. Validation of the oswestry low back pain disability questionnaire, its sensitivity as a measure of change following treatment and its relationship with other aspects of the chronic pain experience. Physiother Theory Pract 1997;13:67-80.

Furlan AD, van Tulder MW, Cherkin DC, Tsukayama H, Lao L, Koes BW, Berman BM. Acupuncture and dry-needling for low back pain. Co- chrane Database Syst Rev 2005;(1):CD001351.

Ganiyu SO, Gujba KF. Effects of acupuncture, core-stability exercises, and treadmill walking exercises in treating a patient with postsurgical lumbar disc herniation: a clinical case report. J Acupunct Meridian Stud 2015;8:48-52

Gattie E, Cleland JA, Snodgrass S. The effectiveness of trigger point dry needling for musculoskeletal conditions by physical therapists: a systematic review and meta-analysis. J Orthop Sports Phys Ther 2017;47: 133-149.

Glaser JA, Baltz MA, Nietert PJ, Bensen CV. Electrical muscle stimulation as an adjunct to exercise therapy in the treatment of nonacute low back pain: a randomized trial. J Pain 2001;2:295-300.

Hicks GE, Sions JM, Velasco TO, Manal TJ. Trunk muscle training augmented with neuromuscular electrical stimulation appears to improve function in older adults with chronic low back pain: a randomized preliminary trial. Clin J Pain 2016;32:898-906.

Jorgensen JE, Afzali T, Riis A. Effect of differentiating exercise guidance based on a patient's level of low back pain in primary care: a mixedmethods systematic review protocol. BMJ Open 2018;8:e19742.

Liu L, Huang QM, Liu QG, Thitham N, Li LH, Ma YT, Zhao JM. Evidence for dry needling in the management of myofascial trigger points associated with low back pain: a systematic review and meta-analysis. Arch Phys Med Rehabil 2018;99:144-152.e2.

Nguyen C, Boutron I, Rein C, Baron G, Sanchez K, Palazzo C, Dupeyron A, Tessier JM, Coudeyre E, Eschalier B, Forestier R, Roques-Latrille CF, Attal Y, Lefèvre-Colau MM, Rannou F, Poiraudeau S. Intensive spa and exercise therapy program for returning to work for low back pain patients: a randomized controlled trial. Sci Rep 2017;7:17956.

Phan NQ, Blome C, Fritz F, Gerss J, Reich A, Ebata T, Augustin M, Szepietowski JC, Ständer S. Assessment of pruritus intensity: prospective study on validity and reliability of the visual analogue scale, numerical rating scale and verbal rating scale in 471 patients with chronic pruritus. Acta Derm Venereol 2012;92:502-507.

Shariat A, Lam ET, Kargarfard M, Tamrin SB, Danaee M. The application of a feasible exercise training program in the office setting. Work 2017; 56:421-428

Shariat A, Noormohammadpour P, Memari AH, Ansari NN, Cleland JA, Kordi R. Acute effects of one session dry needling on a chronic golfer's elbow disability. J Exerc Rehabil 2018;14:138-142.

Shariat A, Tamrin SB, Arumugam M, Danaee M, Ramasamy R. Prevalence rate of musculoskeletal discomforts based on severity level among office workers. Acta Med Bulg 2016;43:54-63.

Takahashi N, Omata JI, Iwabuchi M, Fukuda H, Shirado O. Therapeutic efficacy of nonsteroidal anti-inflammatory drug therapy versus exercise therapy in patients with chronic nonspecific low back pain: a pro- 
spective study. Fukushima J Med Sci 2017;63:8-15.

Tough EA, White AR, Cummings TM, Richards SH, Campbell JL. Acupuncture and dry needling in the management of myofascial trigger point pain: a systematic review and meta-analysis of randomised controlled trials. Eur J Pain 2009;13:3-10.

Triolo RJ, Boggs L, Miller ME, Nemunaitis G, Nagy J, Bailey SN. Implanted electrical stimulation of the trunk for seated postural stability and function after cervical spinal cord injury: a single case study. Arch Phys Med Rehabil 2009;90:340-347.

Tüzün EH, Gıldır S, Angın E, Tecer BH, Dana KÖ, Malkoç M. Effectiveness of dry needling versus a classical physiotherapy program in patients with chronic low-back pain: a single-blind, randomized, con- trolled trial. J Phys Ther Sci 2017;29:1502-1509.

Welsh JT. Assessing pain in the ED including the use of pain scales (such as OSBD, FLACC, VRS, NRS, CRS, and Oucher). Curr Emerg Hosp Med Rep 2016;4:19-25.

Yang YS, Koontz AM, Triolo RJ, Cooper RA, Boninger ML. Biomechanical analysis of functional electrical stimulation on trunk musculature during wheelchair propulsion. Neurorehabil Neural Repair 2009;23: 717-725.

Ye C, Ren J, Zhang J, Wang C, Liu Z, Li F, Sun T. Comparison of lumbar spine stabilization exercise versus general exercise in young male patients with lumbar disc herniation after 1 year of follow-up. Int J Clin Exp Med 2015;8:9869-9875. 